\title{
Reflexiones acerca de la inviabilidad de la demanda laboral como guía orientadora de la planificación universitaria
}

Dr. Ángel R. Velázquez Fernández

Orlando Mirabal González Nérida Rey Córdova

\section{Educación universitaria y su correspondencia con el desarrollo nacional}

No hay dudas acerca de la necesidad de que exista una adecuada correspondencia entre los niveles de desarrollo económico y social. Tanto las tendencias a interpretaciones teóricas, como la propia experiencia práctica, ratifican esa aseveración. Cada paso en el desarrollo económico, cada unidad de crecimiento del Producto Bruto Interno abre nuevas posibilidades para el desarrollo pleno de la sociedad, ampliando la calidad de la vida en todos los órdenes. Al mismo tiempo, este desarrollo social constituye una condición sine qua non del desarrollo económico.

De esta manera, ambos componentes del desarrollo forman una aparente contradicción, ya que los recursos que se destinen a uno de los objetivos limitarán las posibilidades de crecimiento inmediato del otro. Sin embargo, si el análisis se basa en un horizonte temporal más amplio, el desarrollo de una de las esferas es condición indispensable del desarrollo de la otra. En esta contradicción toca a la producción de los bienes materiales jugar el lugar primordial, ya que ella constituye la base económica fundamental de todo el sistema social. Lógicamente, esta supremacía es sólo desde el punto de vista teórico, pues, en la práctica, un desbalance sostenido entre el desarrollo económico y el social, o cualquier intento de priorizar uno en detrimento del otro, tiene como consecuencias negativas a largo plazo. 
La educación universitaria es un reflejo del avance social. A una educación superior desarrollada, debe corresponder una economía también saludable, y viceversa. Los indicadores relativos a la enseñanza del nivel superior, la cantidad de profesionales egresados de las universidades y el contexto académico en que se desenvuelve, son índices indiscutibles de bienestar espiritual, de calidad de vida y de desarrollo económico.

La complejidad de la sociedad moderna y la influencia creciente del progreso de la ciencia y la tecnología, imponen la necesaria correspondencia entre economía y universidad. Es desde todo punto de vista imposible concebir la educación superior de hoy sin tener en cuenta los requerimientos económicos; es imposible una política de desarrollo universitario sin tener una visión clara de lo que espera la sociedad de las universidades; sin que exista una valoración de las tendencias de la economía nacional y sin una visión integradora entre universidad y desarrollo.

Hasta aquí, no hay motivos para cuestionamientos. Ahora bien, el problema radica en cómo interpretar la naturaleza de este vínculo y cómo traducirlo al lenguaje común de las políticas, planes y programas de desarrollo. Veamos cómo se manifiesta en el Perú actual.

La Constitución Política, en su artículo 13, establece: "La educación tiene como finalidad el desarrollo integral de la persona humana. El Estado reconoce y garantiza la libertad de enseñanza".

Asimismo, en su artículo 14, plantea: "La educación promueve el conocimiento, el aprendizaje y la práctica de las humanidades, la ciencia, la técnica, las artes, la educación física y el deporte. Prepara para la vida y el trabajo y fomenta la solidaridad".

En cuanto al nivel superior, la Constitución expresa: "La educación universitaria tiene como fines la formación profesional, la difusión cultural, la creación intelectual y artística y la investigación científica y tecnológica..."

En los artículos anteriores, imprescindibles para el objetivo del presente trabajo - aunque no seamos muy originales según Turnitín - se pone de manifiesto que el espíritu del documento constitucional reconoce al ser humano y su desarrollo integral como fin último del sistema educativo.

El mismo sentido puede apreciarse en la Ley 28044, Ley General de Educación, la cual, en su artículo 2 plantea:

...la educación es un proceso de aprendizaje y enseñanza que se desarrolla a lo largo de toda la vida y que contribuye a la formación integral de las personas, al pleno desarrollo de sus potencialidades, a la creación de cultura, y al desarrollo de la familia y de la comunidad nacional, latinoamericana y mundial...

La cuestión cambia de manera radical en la Ley 30220, la cual define, como fines de la Universidad:

6.1 Preservar, acrecentar y transmitir de modo permanente la herencia científica, tecnológica, cultural y artística de la humanidad. 
6.2Formar profesionales de alta calidad de manera integral y con pleno sentido de responsabilidad social de acuerdo con las necesidades del país.

Mientras que la Constitución y la Ley 28044 la formación del ser humano es el fin, en la Ley Universitaria es un medio pues se supedita a las necesidades del país. Este hecho se pone de manifiesto con mayor claridad en su artículo 27, que a continuación citamos:

\section{Artículo 27. Requisitos para la creación de universidades}

Los requisitos básicos que se deben contemplar en los instrumentos de planeamiento para la creación de una institución universitaria, en cualquiera de los niveles, son los siguientes:

27.1 Garantizar la conveniencia y pertinencia con las políticas nacionales y regionales de educación universitaria.

7.2 Vincular la oferta educativa propuesta a la demanda laboral.

Este artículo se concreta en las condiciones básicas de calidad (CBC) establecidas por la Superintendencia Nacional de Educación Superior Universitaria (SUNEDU), las cuales exigen, en la condición 2, la necesidad de fundamentar la propuesta de creación de un nuevo programa académico o universidad, en un estudio de demanda laboral.

De acuerdo con los criterios anteriores, en corto tiempo desaparecerían las profesiones artísticas y la investigación básica en el Perú. Para las primeras, es ilógico hablar de demanda laboral o de contribución al desarrollo económico del país. La segunda no tiene utilidad inmediata.

Las concepciones detrás de las normas señaladas en los párrafos anteriores están fuertemente emparentadas con las políticas de planificación centralizada de los antiguos países socialistas, cuyo rotundo fracaso en la búsqueda del bienestar por medio de mecanismos controlistas que anteponen "la sociedad" al ser humano, la historia reciente se ha encargado de demostrar.

A continuación, tratamos de plantear algunos argumentos que pretenden demostrar lo errado de estas políticas.

\section{¿DEMANDA ECONÓMICA O SOCIAL?}

A los efectos de estudiar los aspectos relacionados con la correspondencia entre educación y economía, y con el objetivo de facilitar su interpretación por parte de los planificadores encargados de coordinar las políticas de desarrollo a todos los niveles, resulta altamente aceptada, desde el punto de vista metodológico, la división de las necesidades educacionales en dos conceptos: demanda económica y demanda social.

El desarrollo económico de cualquier país requiere de una determinada proporción de recursos humanos con altos niveles de calificación. Esta proporción, a la que se denomina demanda económica, debe tener, en situaciones normales, una tendencia creciente y está condicionada por diferentes factores que van desde la reproducción extensiva del sistema económico hasta la intensificación y elevación de la complejidad científica y tecnológica de los procesos de trabajo. 
Por otra parte, se reconoce la necesidad de que todos los ciudadanos tengan acceso a los conocimientos atesorados por la sociedad a través de su historia, lo cual se logra fundamentalmente a través de la educación. Esta necesidad se enmarca en el objetivo más amplio del desarrollo multifacético de la personalidad de las personas. A las exigencias que se derivan de las aspiraciones de los individuos por alcanzar un determinado tipo y nivel de educación es lo que se conoce como demanda social de educación.

En condiciones de limitación de recursos para la educación, la unidad de la demanda económica y la demanda social deviene en la siguiente interrogante; ¿qué se debe satisfacer prioritariamente la educación superior, la demanda económica o la social? ¿Qué formas debe adoptar la respuesta a cada una de ellas? Está claro que esta pregunta solo tiene sentido para las instituciones públicas, mas no para las privadas que se guían por la lógica del mercado. A continuación, se abordan algunas ideas con relación a estas interrogantes.

Si se tiene en cuenta el contenido, tanto de la demanda económica como la social, se puede interpretar la primera como el límite mínimo a que puede aspirar la sociedad. Este aspecto cubre los requerimientos de fuerza de trabajo calificada que plantea la economía nacional. En este sentido, la cantidad de profesionales egresados de las universidades está respaldada por una cantidad equivalente de puestos de trabajo calificados. Cada estudiante tiene un puesto de trabajo esperando que culmine sus estudios, de lo contrario no tendría sentido la planificación.

A pesar de las dificultades metodológicas que encierra el problema del cálculo de la demanda de profesionales, si ello pudiera lograrse, deberían tenerse en cuenta las siguientes: ¿cómo se debe proceder en períodos de crisis, cuando no existen perspectivas visibles de recuperación económica a corto plazo? ise cierran las universidades o los programas vinculados a los sectores económicos que ya no demandan nuevos profesionales? ¿qué hará la sociedad con los centenares de miles de jóvenes que egresan de la secundaria cada año? ¿los planificadores se han detenido a pensar en la frustración de los jóvenes que no pueden acceder a la universidad, simplemente porqué los cálculos dicen que no hay demanda laboral de la profesión que a ellos les satisface estudiar? Y no menos importante ¿el derecho constitucional a la educación puede restringirse por consideraciones economicistas?

Los que piensan que regular la cantidad de matriculados en las universidades constituye la solución del problema, simplemente están trasladando la insatisfacción de una necesidad (empleo de nivel superior) en el campo de la economía, hacia la insatisfacción de otra (estudios universitarios), en el campo educacional. Por tanto, desde este punto de vista, la regulación y contracción del nivel de ingreso a las universidades no puede catalogarse como un éxito de la planificación, sino como un índice que expresa la incapacidad del sector productivo de absorber nueva fuerza de trabajo calificada. Cabe entonces plantear la siguiente pregunta: ¿Qué es preferible para la sociedad, un profesional sin empleo calificado o un joven cuyo derecho a educarse se le limita a la secundaria, porque no hay demanda laboral?

Apartándonos un poco del problema del empleo, cabe preguntarse: ¿qué significado político y social tiene el hecho de que la sociedad tenga posibilidades de ofrecer sus conocimientos a 
determinada cantidad de jóvenes y no lo haga? ¿Se desconoce que la función principal del sistema educacional es la formación integral del ser humano?

Debe tenerse en consideración, para responder a la interrogante anterior, que lo que realmente determina la continuidad histórica del desarrollo, es precisamente esa capacidad del hombre de traspasar a la generación siguiente toda la experiencia y conocimientos acumulados, capacidad que desde un inicio lo diferenció del resto de las otras especies del reino animal, y que posibilitó que la sociedad sea tal como la vivimos hoy. Aunque esta transmisión de experiencias se realiza también por otros medios, sin duda que esto constituye un proceso muy lento, no apto para el ritmo de las transformaciones que el hombre ha logrado hacer. Para este objetivo la sociedad creó las instituciones educacionales, donde se adquieren los conocimientos y experiencias de una manera directa y relativamente rápida hacia la nueva generación. ¿Debemos limitar este proceso, reduciendo o congelando las vacantes en las aulas universitarias?

Creemos, y esto responde a una pregunta anteriormente formulada, que a la sociedad le resulta mucho más útil un profesional con elevada calificación y cultura que no esté ocupando (quizás temporalmente) un puesto de nivel superior, que dejar de ofrecer la mayor cantidad de conocimientos a las generaciones futuras, al restringir el acceso a las aulas universitarias.

A nuestro juicio, la magnitud del acceso a la educación universitaria debería estar dada, fundamentalmente, por tres factores:

- La disponibilidad de recursos que tiene la sociedad para dedicarlos a la educación superior.

- El comportamiento de las variables demográficas, las cuales pueden ocasionar disminuciones de la población apta para ingresar a la educación superior.

- El comportamiento de los niveles precedentes del sistema educacional, el cual puede incidir en la cantidad de aspirantes con el nivel académico adecuado para el ingreso a las instituciones de educación superior.

Cada uno de estos factores debe jugar un papel de primer orden en la determinación de los volúmenes de ingreso a las universidades y centros de enseñanza superior, y deben constituir, por tanto, las variables fundamentales que deben tenerse en cuenta durante el proceso de planificación.

Como se señaló anteriormente, en condiciones de escasez de recursos y de dificultades económicas, la sociedad debe considerar cuidadosamente cómo emplear estos recursos. Por tanto, la creación de capacidades en la educación superior (en el sector público) deberá estar supeditada a un análisis profundo de los requerimientos de la economía, con vistas a lograr los niveles mínimos de graduados que satisfagan, en primer lugar, la demanda económica y teniendo en cuenta las exigencias relacionadas con la calidad del profesional formado. Se pudiera pensar que una reducción de la cantidad de estudiantes redundaría en una elevación de la calidad del proceso de enseñanza, pero hasta qué punto esto puede justificar limitación del nivel de acceso a las universidades, debe ser objeto de un minucioso estudio.

El comportamiento de las variables demográficas puede limitar el número de candidatos a ingresar a la universidad. El análisis de su evolución proporciona una información de gran importancia 
para la proyección del desarrollo del sistema educacional en su conjunto, en particular del nivel superior. La experiencia internacional muestra cómo, en determinados períodos, el comportamiento poblacional puede mostrar una tendencia a disminuir el número de habitantes en determinados grupos de edades, por lo que el papel de los planificadores debe estar dirigido al análisis de las posibles variantes que conduzcan a una utilización racional de las capacidades existentes durante el período en el cual se producirán afectaciones en los niveles de ingreso a las universidades.

Como es conocido, el desarrollo demográfico y el económico son procesos que, aunque interrelacionados, no tienen un comportamiento cuantitativo sincrónico. Si, por ejemplo, en un punto de alza demográfica en la edad típica de ingreso a la educación superior se produjera una contracción de la demanda económica, esto implicaría una disminución del porcentaje de postulantes que ingresan a la universidad, provocando efectos negativos de carácter social.

En primer lugar, la sociedad renunciaría a satisfacer una necesidad social, contando con los recursos y capacidades requeridas para ello. Por otra parte, puede ocurrir un fenómeno que llamaremos "derecho por coyuntura". Esto significa que el derecho a la educación (el cual tiene carácter general), estará en dependencia de fenómenos coyunturales, ajenos al propio sistema educacional. Manteniendo una demanda económica constante, aquellos que nazcan en épocas de baja tasa de natalidad, tendrán muchas mayores posibilidades de acceso a la enseñanza superior, mientras que, en circunstancias contrarias, se requerirá de un esfuerzo mucho mayor por parte de los educandos. ¿Cuántos talentos puede perder la sociedad por estas razones? ¿Cuántos profesionales menos dotados impedirán la superación y el empleo de individuos más capacitados intelectualmente? ¿Acaso esto no acarrea pérdidas económicas por la simple razón del comportamiento demográfico?

Los resultados obtenidos por el nivel precedente de enseñanza, constituye un tercer factor que incide en el comportamiento del ingreso a la educación superior.

Deficiencias en la calidad de la preparación académica de los estudiantes con posibilidades de acceso a la universidad, pueden limitar e incluso disminuir el número de estudiantes que ingresa realmente a la enseñanza de nivel superior, aunque esto no deberá ser nunca un elemento para reducir "planificadamente" el número de plazas ofertadas. En este sentido, se produce un proceso de "selección natural", a partir del establecimiento de un sistema de ingreso capaz de detectar estas insuficiencias en la formación del estudiante.

En nuestro criterio el aspecto más relevante de la demanda económica de fuerza de trabajo calificada no es el aspecto cuantitativo, sino el cualitativo: su influencia en la definición del tipo de programas que se ofertan y en el contenido de los currículos universitarios. Su influencia cuantitativa en los niveles de acceso a las universidades debería ser observada con recelo, por las razones ya mencionadas.

Un aspecto que generalmente no es tenido en cuenta, lo constituye el hecho de que, en la práctica existen dificultades metodológicas y conceptuales para la realización del cálculo de la demanda económica por sectores de la economía, que hacen imposible lograr resultados serios y confiables, más allá de los planteamientos que puedan realizar los "expertos" en algunas columnas periodísticas u otros medios de comunicación. 


\section{¿Demanda económica?}

De todo lo analizado anteriormente, se desprende que los conceptos de demanda económica y social desempeñan un papel importante en la política de desarrollo de la educación superior, pero, al mismo tiempo, la interpretación y aplicación deformada de los mismos, puede conducir a la toma de decisiones que, a la larga, perjudicarían el desarrollo de la economía nacional y en general de la sociedad. Y en este punto resultará necesaria una mayor reflexión acerca de las limitaciones de la demanda económica como criterio de planificación.

La consideración de la demanda económica como un límite máximo a los niveles de ingreso, lleva aparejado, de hecho, un desconocimiento de una parte importante de las necesidades de educación superior que plantea la sociedad. Es cierto que toda demanda económica satisface al mismo tiempo la demanda social, pero no a la inversa; es decir, toda demanda social no satisface, directamente, la llamada demanda económica, la cual constituye un subconjunto de la primera.

Al aceptar que la demanda económica expresa la correspondencia entre el número de graduados y la cantidad de empleos calificados que genera la economía nacional, y se acude a este concepto como vía para solucionar los problemas que se presentan en la práctica, con el empleo y la utilización de la fuerza de trabajo calificada, se puede aceptar, por simple despeje, que aquella parte de la demanda social que queda fuera de las exigencias puramente económicas, no podrá ser satisfecha nunca en estas condiciones, pues siempre, en cualquier etapa de desarrollo, graduar más profesionales que aquellos que requiere exactamente la economía generará inevitablemente este tipo de problemas de empleo. Por tanto, aceptar la demanda económica como límite máximo es negar la existencia de la necesidad social, incluso desde un plano conceptual.

Si se admite que, en un momento dado, ambas demandas coinciden desde el punto de vista cuantitativo, entonces la división de estos conceptos resultaría superflua y sin sentido de ningún tipo, ya que se estaría dando dos nombres a un mismo fenómeno. Empero, como esta coincidencia no puede ocurrir, el hecho de tomar las exigencias de la economía como límite máximo de las cifras de ingreso, significaría una condena a la eterna insatisfacción de la demanda social.

A esto hay que añadir que la separación entre demanda económica y social tiene valor sólo desde el punto de vista metodológico, pues, en la práctica, ambos conceptos confluyen en el interés, capacidad o vocación de un individuo, ya sea joven estudiante o trabajador, mujer u hombre, empresario, obrero o campesino. Este individuo tomará la decisión que a su juicio resulte más acertada, teniendo en cuenta sus intereses personales y valorando su posibilidad de ser más útil. Para él la contradicción entre demanda económica y social no constituye un motivo evidente para renunciar al acceso a la educación superior.

\section{El cálculo de la demanda económica y sus limitaciones actuales}

Hasta aquí se abordó el problema de las limitaciones que presenta el concepto de la demanda económica, al considerarse como límite máximo para determinar los volúmenes de ingreso a la educación superior, aunque suponiendo siempre que esta demanda resulta cuantitativamente exacta y, por tanto, constituye un fiel reflejo de la necesidad económica de fuerza de trabajo 
calificada. Sin embargo, pueden plantearse las siguientes interrogantes: ¿están suficientemente fundamentados desde, el punto de vista metodológico, estos cálculos, como para tomar decisiones que limiten el acceso a la universidad en los próximos años?

Esta interrogante requiere una respuesta que, por su magnitud y complejidad, se sale de los marcos de este artículo.

Independientemente de las posibles limitaciones derivadas de la metodología que pudiera emplearse, los argumentos más importantes utilizados para cuestionar la validez del cálculo de la demanda económica son: la propia debilidad del concepto de demanda económica, como elemento determinante y punto de partida para la planificación de la educación superior; la naturaleza de la economía de mercado, con fluctuaciones cíclicas e impredecibles para la mayoría de las variables económicas y la falta de visión prospectiva acerca del desarrollo futuro de gran número de empresas e instituciones.

Por último, los estudios de demanda son incapaces de pronosticar ni siquiera de manera aproximada, las nuevas ocupaciones que surgirán a mediano plazo, las tecnologías que transformarán industrias completas en menos de un lustro, las nuevas competencias que requerirán los trabajadores. Responder a esas demandas solo pueden hacerlo las universidades en el marco de su autonomía y en pleno ejercicio de sus capacidades, no los burócratas de la planificación. Recordemos que, a mediados de los años ochenta del siglo pasado, muchos teóricos estaban convencidos que el futuro del mundo era el socialismo, hoy ellos mismos tienen la certeza de que el futuro puede ser cualquier sorpresa, menos socialista.

\section{CONSIDERACIONES FINALES}

A partir de los elementos tratados anteriormente, se pueden ofrecer las siguientes consideraciones:

- La educación superior tiene como función principal brindar la posibilidad de desarrollar todas las capacidades de los individuos. De aquí que no se debe restringir a priori la demanda por razones economicistas (demanda económica). El efecto de esta última debe reducirse a ser el límite mínimo del ingreso en las instituciones públicas, orientar a los jóvenes que optan por seguir estudios universitarios y servir de referencia para la definición del contenido de los planes de estudio de los perfiles profesionales.

- No existen mecanismos de planificación idóneos que permitan pronosticar la demanda económica de fuerza de trabajo calificada a largo plazo y un profesional tiene una vida útil de más de cuarenta años, tiempo más que suficiente para que surjan y desaparezcan profesiones, oficios y sectores económicos completos.

- La organización del trabajo en la sociedad se adapta de manera permanente a la existencia de personas altamente calificadas a escala social. Esto presupone que la estructura del empleo no debe ser traba o un freno al desarrollo intelectual de los individuos. Debe, por tanto, eliminarse cualquier concepción que atente contra este objetivo. Ha de partirse del principio de que en la sociedad existe tanta o más necesidad de trabajo calificado como ella es capaz de formar, por lo que la organización del trabajo no puede inhibir la plena utilización de este potencial. 
- Debe quedar claramente definido que los problemas que surgen en el campo del empleo no pueden ser totalmente resueltos en el terreno educacional y mucho menos restringiendo el crecimiento de las nuevas plazas ofertadas por la educación superior, debido a que el sistema educacional cumple otras importantes funciones que no pueden ser soslayadas ni relegadas a un segundo plano.

- Es interés de este trabajo promover un debate acerca de las trabas y limitaciones a la creación de nuevos programas, así como de los peligros que tiene una política dirigida, única y exclusivamente a la satisfacción de la demanda económica. De lograrlo, los autores habrán alcanzado sus objetivos.

\section{REFERENCIAS}

Constitución Política del Perú. 1993

Ley 28044, Ley General de Educación. 2003

Ley 30220, Ley Universitaria. 2014

SUNEDU (2017) Manual de Instrucciones para la presentación de la solicitud de licenciamiento y de la modificación del licenciamiento institucional 